\title{
Unrecognized History of Transient Atrial Fibrillation at the Time of Discharge from an Index Stroke Hospitalization Is Associated with Increased Recurrent Stroke Risk
}

\author{
Chia-Yu Hsu, ${ }^{\mathrm{a}}$ Daniel E. Singer, ${ }^{\mathrm{b}}$ Hooman Kamel, ${ }^{\mathrm{c}}$ Yi-Ling Wu, ${ }^{\mathrm{d}}$ Pei-Chun Chen, ${ }^{\mathrm{e}}$ Jiann-Der Lee, ${ }^{\mathrm{a}}$ \\ Meng Lee, ${ }^{\mathrm{a}}$ Bruce Ovbiagele ${ }^{\mathrm{f}}$ \\ aDepartment of Neurology, Chang Gung Memorial Hospital, Chang Gung University College of Medicine, Chiayi, Taiwan \\ ${ }^{b}$ Division of General Internal Medicine, Massachusetts General Hospital, Harvard Medical School, Boston, MA, USA \\ 'Department of Neurology, Weill Cornell Medical College, New York, NY, USA \\ ${ }^{d}$ National Health Research Institutes, Institute of Population Health Sciences, Miaoli, Taiwan \\ 'Department of Public Health, China Medical University, Taichung, Taiwan \\ fDepartment of Neurology, Medical University of South Carolina, Charleston, SC, USA
}

Background and Purpose Preceding episodes of paroxysmal atrial fibrillation (AF) among stroke patients can be easily overlooked in routine clinical practice. We aim to determine whether an unrecognized history of paroxysmal AF is associated with an increased risk of recurrent stroke.

Methods We retrospectively identified all adult patients hospitalized with a primary diagnosis of ischemic stroke who had no AF diagnosis on their discharge records, using the Taiwan National Health Insurance Research Database between January 2001 and December 2012. Patients were categorized into two groups: unrecognized AF history and no AF. Patients with unrecognized AF history were defined as having documented AF preceding the index ischemic stroke hospitalization, but not recording at the index ischemic stroke. Primary endpoint was recurrent stroke within 1 year after the index stroke.

Results Among 203,489 hospitalized ischemic stroke patients without AF diagnosed at discharge, 6,731 patients (3.3\%) had an unrecognized history of prior transient AF. Patients with an unrecognized AF history, comparing to those without $A F$, had higher adjusted risk of all recurrent stroke ([original cohort: hazard ratio (HR), 1.41; 95\% confidence interval [Cl], 1.30 to 1.53], [matched cohort: $\mathrm{HR}, 1.51 ; 95 \% \mathrm{Cl}, 1.37$ to 1.68$]$ ]) and recurrent ischemic stroke ([original cohort: $\mathrm{HR}, 1.42 ; 95 \% \mathrm{Cl}, 1.30$ to 1.55 ], [matched cohort: $\mathrm{HR}, 1.56 ; 95 \% \mathrm{Cl}, 1.40$ to 1.74 ]) during the 1 -year follow-up period.

Conclusions Unrecognized history of AF among patients discharged after an index ischemic stroke hospitalization is associated with higher recurrent stroke risk. Careful history review to uncover a paroxysmal AF history is important for ischemic stroke patients.
Correspondence: Meng Lee Department of Neurology, Chang Gung Memorial Hospital, Chang Gung University College of Medicine, No. 6, Sec W, Jiapu Rd, Puzi, Chiayi 613, Taiwan Tel: $+886-5-3621000$

Fax: +886-5-3623002

E-mail: menglee5126@gmail.com

Received: November 19, 2018 Revised: February 14, 2019 Accepted: February 15, 2019

Keywords Atrial fibrillation; Medical records; Brain infarction

Copyright @ 2019 Korean Stroke Society

This is an Open Access article distributed under the terms of the Creative Commons Attribution Non-Commercial License (http://creativecommons.org/licenses/by-nc/4.0/) which permits unrestricted non-commercial use, distribution, and reproduction in any medium, provided the original work is properly cited. 


\section{Introduction}

Atrial fibrillation (AF) is a modifiable risk factor of ischemic stroke, and $20 \%$ to $30 \%$ of ischemic stroke patients have AF diagnosed before, during, or after stroke events. ${ }^{1}$ In patients with $A F$, more than $60 \%$ of strokes could be prevented by use of an oral anticoagulant (OAC). ${ }^{2,3}$ For ischemic stroke patients with permanent or paroxysmal $A F$, an $O A C$ is generally indicated to prevent recurrent strokes. ${ }^{4}$ However, in real-world practice, reports have shown that a substantial proportion of AF patients who are eligible for OAC treatment remained untreated or inadequately treated by antiplatelet agents. ${ }^{5}$

Both permanent and paroxysmal AF are related to increased stroke risk and warrant anticoagulants treatment, but paroxysmal AF especially makes a big challenge in clinical practice. ${ }^{6}$ Some stroke patients may not have AF found on 12-lead electrocardiogram or even long-term cardiac monitoring during an index stroke hospitalization, but may have a history of transient paroxysmal AF. Transient paroxysmal AF may be easily overlooked if it is not clearly communicated by the patient or not clearly documented in accessible medical records.

We hypothesized that the ischemic stroke patients who had an untreated transient paroxysmal AF history that was overlooked and remained untreated during the index stroke hospitalization may have an increased recurrent stroke risk, as compared to stroke patients without AF. We performed this study to determine whether an unrecognized history of paroxysmal AF increased the 1-year recurrent stroke risk among ischemic stroke patients without AF identified at discharge.

\section{Methods}

We conducted a retrospective nationwide cohort study using the Taiwan National Health Insurance Research Database (NHIRD) in the period from January 2001 to December 2012, using the 9th revision of the International Classification of Diseases (ICD-9). Inclusion criteria were: (1) patient $\geq 20$ years old, (2) first episode of stroke hospitalization with a primary diagnosis of ischemic stroke (ICD-9 codes 433.X1, 434.X1, 436) in the study period, and (3) patient survived and had no AF diagnosis (ICD-9 code 42731) at discharge. Stroke hospitalization was defined as admission for inpatient service in any department in medical institutions, not including outpatient clinics or emergency department visits. Any stroke hospitalization with admission date more than 3 days apart from discharge date of a previous stroke hospitalization was defined as a new stroke hospitalization. We excluded patients who had no computed tomography or magnetic resonance imaging of brain during the index hospitalization or within 7 days prior to admission, because the diagnosis of ischemic stroke may be inaccurate without the confirmation of brain imaging. We also excluded patients who had used anticoagulant during the index hospitalization, because these patients may have recognized AF that was not recorded in the first five diagnoses in the discharge medical records. We further excluded patients with diagnosis of intracranial hemorrhage (ICD-9 codes $430 . x x-432 . x x)$, gastrointestinal hemorrhage (ICD-9 codes 578.xx), or hematuria (ICD-9 codes 599.70) before or during index stroke hospitalization, because these patients may be untreated with anticoagulants because they had history of major bleeding events, but not because their AF were "unrecognized." Prior diagnosis of AF was ascertained looking back from the stroke date to the beginning of 1997 since we used data starting from 1997. The median of the look-back period for an AF diagnosis was 11.2 years (interquartile range, 8.9 to 13.5). Included patients were categorized into two groups: unrecognized AF history and no AF. Patients with unrecognized AF history were defined as having had an AF diagnosis at least once during any hospitalization preceding the index stroke hospitalization or at least twice in the outpatient setting before the index stroke (Figure 1).

The primary endpoint was any recurrent stroke (ICD-9 430$434,436)$, and secondary endpoints were any recurrent ischemic stroke (ICD-9 433.X1, 434.X1, 436), intracranial hemorrhage (ICD-9 430-432), and AF (ICD-9 427.31) within 1 year after discharge from the index stroke.

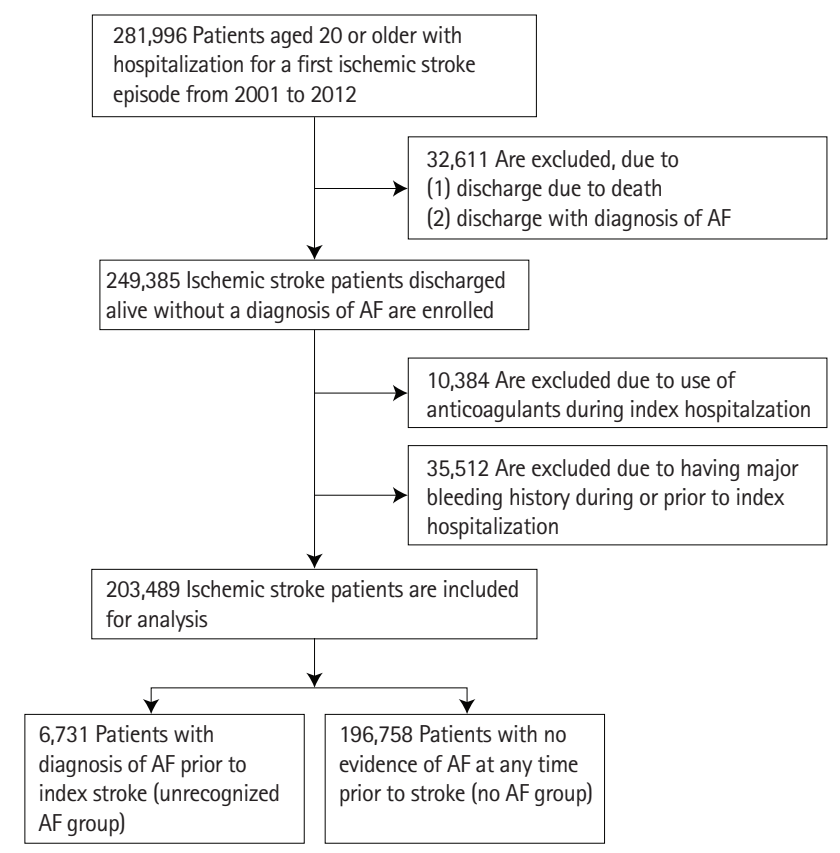

Figure 1. A flowchart of study process. AF, atrial fibrillation. 
This study was approved by the Institutional Review Board of Chang Gung Memorial Hospital, Chiayi, Taiwan. This was a nationwide study that included all available and eligible patients. The individual's personal information in the databases were encrypted for privacy protection; therefore, the requirement of informed consent was waived.

Comorbidities before the index stroke for each case were extracted, including hypertension (ICD-9 401-405), ischemic heart disease (ICD-9 410-414), diabetes mellitus (ICD-9 250), dyslipidemia (ICD-9 272), prior stroke (ICD-9 430-434, 436438), heart failure (ICD-9 428), peripheral vascular disease (ICD-9 443.9), chronic obstructive pulmonary disease (ICD-9 491, 492, 496), sleep apnea (ICD-9 327.23, 780.57), and chronic kidney disease (ICD-9 585, 403). Stroke severity was evaluated by the stroke severity index (SSI) (airway suctioning, bacterial sensitivity test, general ward stay, intensive care unit stay, nasogastric intubation, osmotherapy, and urinary catheterization), developed specifically to evaluate the severity of strokes in Taiwan NHIRD.?

\section{Statistical analysis}

To mitigate differences of baseline characteristics between the two groups (unrecognized AF vs. no AF), we conducted propensity score matching. We performed logistic regression models for variables including sex, age, SSI, hypertension, ischemic heart disease, diabetes mellitus, dyslipidemia, prior stroke, heart failure, peripheral vascular disease, chronic obstructive pulmonary disease, sleep apnea, and chronic kidney disease for propensity score analysis and created a 1:2 matched pairs. The baseline characteristics of original and matched two groups were compared using standardized difference (SD). SD $>0.1$ was considered as statistically significant difference between the two groups.

We employed the Cox regression for original cohort and stratified Cox regression for matched cohort to estimate the unadjusted and adjusted hazard ratios (HRs) and 95\% confidence intervals (Cls) of the group having unrecognized history of transient $A F$, with the group having no AF history as the reference. The model was adjusted for baseline age, sex, hypertension, diabetes mellitus, hyperlipidemia, ischemic heart disease, stroke history, chronic kidney disease, heart failure, chronic obstructive pulmonary disease, peripheral vascular disease, sleep apnea, and SSI. A 2-sided $P<0.05$ were considered to be statistically significant.

The data were analyzed with SAS statistical software version 9.4 (SAS Institute Inc., Cary, NC, USA).

\section{Results}

Among 203,489 ischemic stroke patients discharged alive without an AF diagnosis at discharge, 6,731 patients (3.3\%) had an unrecognized history of transient AF before index stroke. In original cohort, patients with unrecognized history of transient AF were older, less likely to be men, more often having hypertension, ischemic heart disease, prior stroke history, chronic kidney disease, heart failure history, chronic obstructive pulmonary disease, less often having hyperlipidemia, and having more severe stroke symptoms than patients without AF (Table 1). In matched cohort, the age, sex, stroke severity and comorbidities in the two groups had no significant difference (Table 1).

During the 1-year follow-up period, patients with unrecognized AF history had higher risks of all recurrent stroke ([original cohort: $\mathrm{HR}, 1.41 ; 95 \% \mathrm{Cl}, 1.30$ to 1.53], [matched cohort: $H R, 1.51 ; 95 \% \mathrm{Cl}_{1} 1.37$ to 1.68$\left.]\right)$, recurrent ischemic stroke ([original cohort: $\mathrm{HR}, 1.42 ; 95 \% \mathrm{Cl}, 1.30$ to 1.55 ], [matched cohort: $\mathrm{HR}, 1.56 ; 95 \% \mathrm{Cl}, 1.40$ to 1.74$]$ ), and newly-detected $\mathrm{AF}$ ([original cohort: $\mathrm{HR}, 11.3 ; 95 \% \mathrm{Cl}, 10.7$ to 12.0$]$, [matched cohort: $\mathrm{HR}, 9.66 ; 95 \% \mathrm{Cl}, 8.84$ to 10.55$]$ ) after adjustment of relevant covariates. There was a non-significant trend of increased risk for hemorrhagic stroke ([original cohort: HR, 1.29; 95\% Cl, 0.96 to 1.72], [matched cohort: HR, 1.10; 95\% Cl, 0.79 to 1.54]), when compared to patients without AF (Table 2). For all endpoints, there is no difference in stratified Cox regression.

Before the index stroke, 907 of patients (13.5\%) with unrecognized AF and 1,909 patients (1\%) with no AF had used anticoagulants. After discharge from the index stroke, 859 of patients (14.76\%) with unrecognized AF and 243 of patients (1.85\%) with no AF were prescribed anticoagulants.

\section{Discussion}

This nationwide study showed that among ischemic stroke patients without AF diagnosed at discharge, 3.7\% actually had an unrecognized history of transient $A F$, and these patients had higher risks of recurrent strokes, recurrent ischemic strokes, and $\mathrm{AF}$ at 1-year follow-up compared to ischemic stroke patients without $A F$.

OACs prevent primary strokes in AF patients and prevent recurrent strokes in ischemic stroke patients with AF. ${ }^{1}$ However, $25 \%$ to $40 \%$ of AF is paroxysmal and clinically silent. ${ }^{8}$ As such it can be a major challenge to detect paroxysmal AF in ischemic stroke patients. Our study shows that some hospitalized ischemic stroke patients may have previous history of untreated transient paroxysmal AF that remains unrecognized at the time of hospital discharge, thereby exposing these patients to 
Table 1. Baseline characteristics of original and matched groups without AF and with unrecognized AF

\begin{tabular}{|c|c|c|c|c|c|c|}
\hline \multirow[b]{2}{*}{ Demographic characteristic } & \multicolumn{3}{|c|}{ Original } & \multicolumn{3}{|c|}{ Matched } \\
\hline & $\begin{array}{l}\text { Unrecognized AF } \\
\quad(n=6,731)\end{array}$ & $\begin{array}{c}\text { No AF } \\
(n=196,758)\end{array}$ & $\begin{array}{l}\text { Standardized } \\
\text { difference }\end{array}$ & $\begin{array}{l}\text { Unrecognized AF } \\
\quad(n=6,727)\end{array}$ & $\begin{array}{c}\text { No AF } \\
(n=13,454)\end{array}$ & $\begin{array}{c}\text { Standardized } \\
\text { difference }\end{array}$ \\
\hline Male sex & $3,481(51.7)$ & $116,644(59.3)$ & 0.1527 & $3,479(51.7)$ & 6,969 (51.8) & 0.0016 \\
\hline Age (yr) & $76.9 \pm 9.7$ & $67.8 \pm 12.7$ & 0.7982 & $76.8 \pm 9.7$ & $77.1 \pm 9.6$ & 0.0302 \\
\hline \multicolumn{7}{|l|}{ Comorbidity } \\
\hline Hypertension & $6,035(89.7)$ & $158,660(80.6)$ & 0.2558 & $6,031(89.7)$ & 12,219 (90.8) & 0.0393 \\
\hline Diabetes & $3,024(44.9)$ & $87,218(44.3)$ & 0.0120 & $3,024(45.0)$ & $6,061(45.1)$ & 0.0019 \\
\hline Hyperlipidemia & $2,618(38.9)$ & $89,500(45.5)$ & -0.1338 & 2,618 (38.9) & $5,442(40.5)$ & 0.0313 \\
\hline Ischemic heart disease & $4,755(70.6)$ & $58,901(29.9)$ & 0.8914 & $4,751(70.6)$ & $9,843(73.2)$ & 0.0564 \\
\hline $\begin{array}{l}\text { Prior stroke/transient ischemic at- } \\
\text { tacks }\end{array}$ & $2,072(30.8)$ & $36,662(18.6)$ & 0.2845 & $2,070(30.8)$ & $4,251(31.6)$ & 0.0178 \\
\hline Chronic kidney disease & $844(12.5)$ & $11,350(5.8)$ & 0.2364 & $842(12.5)$ & $1,513(11.3)$ & 0.0393 \\
\hline Heart failure & $3,316(49.3)$ & $18,204(9.3)$ & 0.9793 & $3,312(49.1)$ & $6,401(47.6)$ & 0.0332 \\
\hline $\begin{array}{l}\text { Chronic obstructive pulmonary dis- } \\
\text { ease }\end{array}$ & $2,614(38.8)$ & $36,683(18.6)$ & 0.4577 & $2,610(38.8)$ & $5,216(38.8)$ & 0.0006 \\
\hline Peripheral vascular disease & $213(3.2)$ & $4,156(2.1)$ & 0.0657 & $212(3.24)$ & $346(2.6)$ & 0.0348 \\
\hline Sleep apnea & $22(0.3)$ & $504(0.3)$ & 0.0131 & $22(0.3)$ & $38(0.3)$ & 0.0081 \\
\hline \multicolumn{7}{|l|}{ Stroke severity index } \\
\hline Mild & $4,026(57.4)$ & 155,745 (79.2) & -0.4296 & $4,026(59.9)$ & $8,267(61.5)$ & 0.0327 \\
\hline Moderate & $1,071(16.5)$ & $24,401(12.4)$ & 0.1008 & $1,071(15.9)$ & $2,109(15.7)$ & 0.0067 \\
\hline Severe & $1,634(26.1)$ & $16,612(8.4)$ & 0.4382 & $1,630(24.2)$ & $3,078(22.9)$ & 0.0319 \\
\hline
\end{tabular}

Values are presented as number (\%) or mean \pm standard deviation.

$\mathrm{AF}$, atrial fibrillation.

Table 2. $\mathrm{HR}^{*}$ of primary and secondary outcomes at 1 year in unrecognized AF vs. no AF groups

\begin{tabular}{|c|c|c|c|c|}
\hline Variable & Crude HR (95\% Cl) & $P$ & Adjusted HR (95\% Cl) & $P$ \\
\hline \multicolumn{5}{|l|}{ Original cohort } \\
\hline Recurrent stroke & $1.53(1.42-1.66)$ & $<0.001$ & $1.41(1.30-1.53)^{+}$ & $<0.001$ \\
\hline \multicolumn{5}{|l|}{ Secondary outcome } \\
\hline Ischemic stroke & $1.54(1.42-1.68)$ & $<0.001$ & $1.42(1.30-1.55)^{+}$ & $<0.001$ \\
\hline Intracranial hemorrhage & $1.40(1.06-1.84)$ & 0.018 & $1.29(0.96-1.72)^{+}$ & 0.089 \\
\hline AF documented within 1 year of index stroke & $21.30(21.20-23.45)$ & $<0.001$ & $11.32(10.67-12.02)^{+}$ & $<0.001$ \\
\hline \multicolumn{5}{|l|}{ Matched cohort } \\
\hline Recurrent stroke & $1.50(1.35-1.66)$ & $<0.001$ & $1.51(1.37-1.68)$ & $<0.001$ \\
\hline \multicolumn{5}{|l|}{ Secondary outcome } \\
\hline Ischemic stroke & $1.55(1.39-1.72)$ & $<0.001$ & $1.56(1.40-1.74)$ & $<0.001$ \\
\hline Intracranial hemorrhage & $1.11(0.79-1.55)$ & 0.558 & $1.10(0.79-1.54)$ & 0.581 \\
\hline AF documented within 1 year of index stroke & $9.63(8.81-10.52)$ & $<0.001$ & 9.66 (8.84-10.55) & $<0.001$ \\
\hline
\end{tabular}

$\mathrm{HR}$, hazard ratio; $\mathrm{AF}$, atrial fibrillation; $\mathrm{Cl}$, confidence interval.

*We employed the Cox regression for original cohort and stratified Cox regression for matched cohort to estimate the crude and adjusted HR and 95\% Cls of the group having unrecognized history of transient AF, with the group having no AF history as the reference; ${ }^{\dagger}$ Model was adjusted for age, sex, hypertension, diabetes, hyperlipidemia, ischemic heart disease, prior stroke, chronic kidney disease, heart failure, chronic obstructive pulmonary disease, peripheral vascular disease, sleep apnea, and stroke severity index. 
significantly higher future risk of recurrent stroke. For these patients, use of antiplatelet drugs would be less effective than anticoagulants in the prevention of recurrent strokes.

In Taiwan, there are some situations in that transient AF may be diagnosed but not treated with anticoagulants. First, when the transient AF is diagnosed when the patient has no or only one vascular risk factor. Second, when the patient has plan to do surgery or invasive procedure recently. Third, when the patient has recent bleeding events or major bleeding history. Fourth, when AF is diagnosed in emergency department or in routine health examination, and the patient is not referred to cardiovascular or other adequate outpatient department for follow-up and further management.

There are some possible causes of unrecognized AF history among discharged ischemic stroke patients. First, for patients with an index ischemic stroke mechanism presumed to be noncardioembolic, and a normal electrocardiogram during that hospitalization, the possibility of pre-admission history of transient AF may be overlooked. Second, without the ready availability of preceding outpatient medical record information, clinicians may be unaware of an untreated pre-admission transient episode(s) of AF. This issue may be especially challenging within uncoordinated healthcare systems where patients are hospitalized at tertiary care facilities that are outside of their routine primary health care delivery network.

We suggest that once AF has been detected it should be integrated into a patient's electronic health profile and an autonomic alert system could be activated in certain key clinical scenarios, such as stroke. Moreover, the evaluation of possible pre-admission AF history could be included as an item on a checklist of discharge preparation following an ischemic stroke hospitalization.

There were some limitations. Most importantly, we relied on ICD-9-CM to ascertain AF, and it is possible that some cases of unrecognized AF represented recognized but simply undocumented AF. However, most of these patients would receive anticoagulant treatments, and we had excluded the patients who received anticoagulants during the index hospitalization or for more than 30 days before the index hospitalization. Second, we did not know the specific ischemic stroke subtypes of these patients. Also, we were unable to analyze the possible reasons for why prior AF history remained unrecognized at the time of discharge from an ischemic stroke hospitalization because of the limitations of the insurance database.

\section{Conclusions}

In conclusion, an unrecognized history of transient AF was associated with higher risks of recurrent stroke among ischemic stroke patients. Careful history review to uncover a paroxysmal AF history are important for ischemic stroke patients.

\section{Disclosure}

The authors have no financial conflicts of interest.

\section{References}

1. Kirchhof $P$, Benussi $S$, Kotecha $D$, Ahlsson $A$, Atar D, Casadei $B$, et al. 2016 ESC guidelines for the management of atrial fibrillation developed in collaboration with EACTS. Eur Heart J 2016;37:2893-2962.

2. Hart RG, Pearce LA, Aguilar MI. Meta-analysis: antithrombotic therapy to prevent stroke in patients who have nonvalvular atrial fibrillation. Ann Intern Med 2007;146:857-867.

3. Ruff CT, Giugliano RP, Braunwald E, Hoffman EB, Deenadayalu N, Ezekowitz MD, et al. Comparison of the efficacy and safety of new oral anticoagulants with warfarin in patients with atrial fibrillation: a meta-analysis of randomised trials. Lancet 2014;383:955-962.

4. Kernan WN, Ovbiagele B, Black HR, Bravata DM, Chimowitz $M I$, Ezekowitz MD, et al. Guidelines for the prevention of stroke in patients with stroke and transient ischemic attack: a guideline for healthcare professionals from the American Heart Association/American Stroke Association. Stroke 2014:45:2160-2236.

5. Freedman B, Potpara TS, Lip GY. Stroke prevention in atrial fibrillation. Lancet 2016;388:806-817.

6. Friberg L, Hammar N, Rosenqvist M. Stroke in paroxysmal atrial fibrillation: report from the Stockholm Cohort of Atrial Fibrillation. Eur Heart J 2010;31:967-975.

7. Sung $\mathrm{SF}$, Hsieh $\mathrm{CY}$, Lin $\mathrm{HJ}$, Chen YW, Chen $\mathrm{CH}$, Kao Yang YH, et al. Validity of a stroke severity index for administrative claims data research: a retrospective cohort study. BMC Health Serv Res 2016;16:509.

8. Chiang $C E$, Naditch-Brûlé L, Murin J, Goethals $M$, Inoue $H_{\text {, }}$ O'Neill J, et al. Distribution and risk profile of paroxysmal, persistent, and permanent atrial fibrillation in routine clinical practice: insight from the real-life global survey evaluating patients with atrial fibrillation international registry. Circ Arrhythm Electrophysiol 2012;5:632-639. 